\title{
MS-EMC vs. NEGF: A Comparative Study Accounting for Transport Quantum Corrections
}

\author{
C. Medina-Bailon ${ }^{\ddagger *}$, C. Sampedro ${ }^{\dagger}$, J.L. Padilla ${ }^{\dagger}$, A. Godoy ${ }^{\dagger}$, L. Donetti ${ }^{\dagger}$, F. Gamiz ${ }^{\dagger}$ and A. Asenov ${ }^{\ddagger}$ \\ $\dagger$ Nanoelectronics Research Group, Universidad de Granada, 18071 Granada, Spain. \\ $\ddagger$ School of Engineering, University of Glasgow, Glasgow G12 8LT, Scotland, UK. \\ * e-mail: Cristina.MedinaBailon@glasgow.ac.uk
}

\begin{abstract}
As electronic devices approach the nanometer scale, quantum transport theories have been recognized as the best option to reproduce their performance. Other possible trend, mainly focused on reducing the computational effort, is the inclusion of quantum effects in semi-classical simulators. This work presents a comparison between a NEGF simulator and a MS-EMC tool including S/D tunneling both applied on a DGSOI transistor.
\end{abstract}

Index Terms-Multi-Subband Ensemble Monte Carlo; NonEquilibrium Green Function; direct Source-to-Drain tunneling; quantum effects; DGSOI.

\section{INTRODUCTION}

Short-channel effects (SCEs) degrade the performance of conventional devices as the dimensions are scaled down. From a modeling point of view, phenomena that have not played an important role in previous technological nodes have to be included [1], [2] in order to explain the electrical behavior of aggressively scaled nanodevices. This is the case of quantum transport phenomena and, in particular, Source-to-Drain tunneling (S/D tunneling) that allows electrons to tunnel from the source to the drain through the narrow potential barrier, eroding the gate control. This effect has been presented as a scaling limit in ballistic non-equilibrium Green's Function (NEGF) calculations [3] distorting the MOSFET operation at transistor channel lengths around $3 \mathrm{~nm}$ [4]. However, this phenomenon cannot be straightforward included in semi-classical transport models. Multi-Subband Ensemble Monte Carlo (MSEMC) simulators provide a detailed description of quantum effects in confinement directions and their impact on scattering but, due to the semi-classical MC transport framework, the tunneling phenomena have to be included in a separate way. In this work, S/D tunneling has been implemented in a MS-EMC simulator and compared to ballistic Non-Equilibrium Green's Function results which consider implicitly tunneling in order to assess the MS-EMC implementation.

\section{Methodology}

The simulation framework is based on a MS-EMC code with already demonstrated capabilities in different scenarios [5], [6]. The tool, based on the mode-space approach of quantum transport [7], solves the Schrödinger equation in the confinement direction, and the Boltzmann Transport Equation (BTE) in the transport plane (Figure 1). The system is coupled by solving Poisson equation in the whole simulation domain.

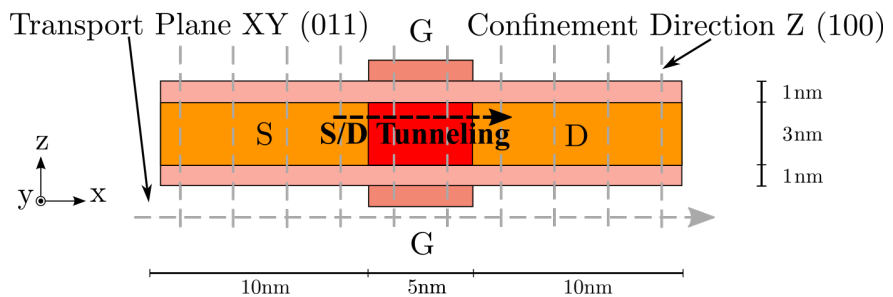

Fig. 1. DGSOI structure analyzed in this work with $\mathrm{L}_{G}$ ranging down to 5 $\mathrm{nm}$ and $\mathrm{T}_{S i}=3 \mathrm{~nm}$. 1D Schrödinger equation is solved for each grid point in the transport direction and BTE is solved by the MC method in the transport plane.

The additional modules needed for taking into account the tunneling are included as separated transport mechanisms and can be activated or not depending on the simulation scenario. S/D tunneling [8] and gate tunneling [9] are implemented as stochastic mechanisms evaluated for each superparticle at the end of Monte Carlo free flight. Band-to-Band tunneling can be also considered as described in [10].

The model used here for the S/D-tunneling extends the non-local band-to-band tunneling algorithm presented in [11] In the present work, the tunneling path and probabilities are calculated considering the starting and finishing points in the corresponding subband of the electron. As in the MSEMC simulator the motion of an electron is studied in the transport direction over a random flight time, it is necessary to determine the the position after the drift process In a semiclassical approximation if the total energy of an electron is smaller than the minimum of the corresponding subband at this position, it will rebound from the potential barrier in the point where the electron and subband energies are the same. In the case of S/D tunneling, electrons may tunnel through the barrier. Therefore, it is necessary to determine tunnel probability and the tunneling path to determine the final position after the flight. The tunneling probability of the electron $T_{d t}$ is calculated using the WKB approximation [12]:

$$
T_{d t}(E)=\exp \left\{-\frac{2}{\hbar} \int_{a}^{b} \sqrt{2 m_{t r}^{*}\left(E_{i}(x)-E\right)} \mathrm{d} x\right\}
$$

Where $a$ and $b$ are the starting and ending points, $E$ and 
$m_{t r}^{*}$ are the energy and transport effective mass of the electron respectively and $E_{i}(x)$ the energy of the corresponding subband. Thanks to the detailed description of the subband structure provided by the MS-EMC simulator, $T_{d t}$ is calculated for each electron considering the minimum of the energy of its subband instead of the Conduction Band [13].

A rejection technique is used to determine whether a electron tunnels or rebound when reaches the barrier. In the case of a tunneling event, the electron will go through the barrier drifted in a ballistic way. Therefore, it is necessary to find the most probable tunneling path. If we assume that all the electrons reach the potential barrier in the starting point with normal direction, the tunneling path can be easily found considering Newton mechanics in the inverted potential $V(\vec{r}) \rightarrow-V(\vec{r})$ and energy $E \rightarrow-E$ [14]. In this system, the potential barrier becomes a valley and electrons go through it undergoing the electric field obtained from this new potential profile in a conservative field.

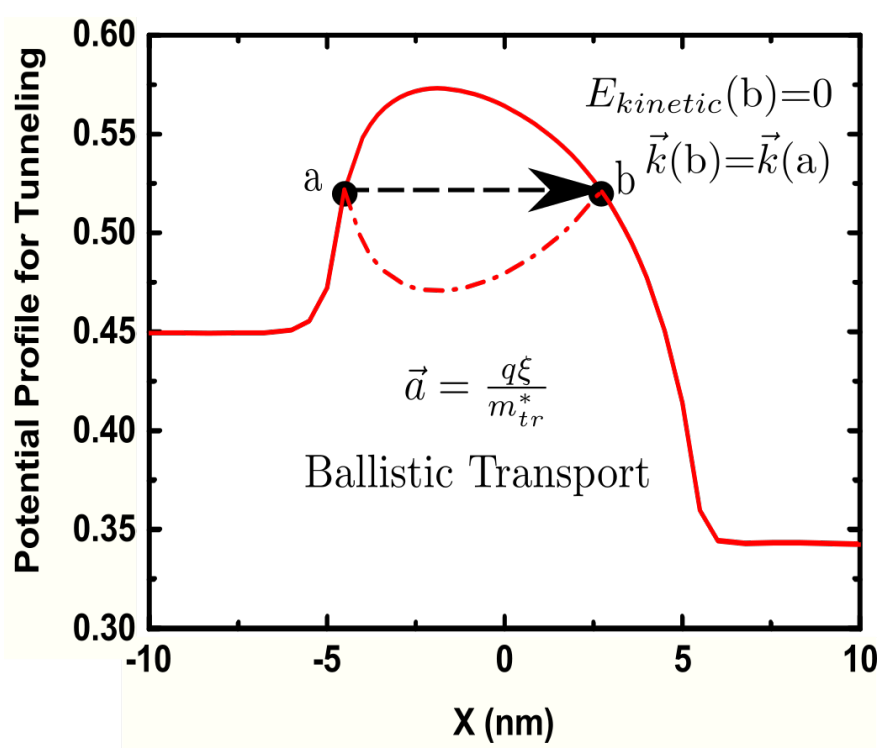

Fig. 2. Representation of the tunneling model: The potential barrier is inverted (dash-dot line) and the particle follows a classical path obeying Newton's second law of motion.

The motion is described as follows,Figure 2. Firstly, an imaginary particle is placed at the starting point $a$ with zero kinetic energy. Before starting its tunneling path, the angle, which determines the relationship between $k_{x}$ and $k_{y}$ and, as a consequence, its flight direction, is maintained. Then, it enters the tunneling region where it is accelerated in obeying Newton's second law of motion in the inverted potential profile (dash-dot line). Finally, it appears at some point $b$ in the minimum of its subband again with zero kinetic energy. That particle continues to flow into the device with the same transport properties.

In order to assess the accuracy of including quantum transport in this way, the simulation results will be compared to those obtained with NanoMOS [15], a Non-Equilibrium Green's Function (NEGF) 2D simulator for ultrathin DGSOI devices that considers ballistic transport in an effective mass approximation.

\section{RESUlTS AND DISCUSSION}

Figure 1 shows the double gate structure and device parameters herein analyzed. In this study, the gate length ranges from $L_{G}=10 \mathrm{~nm}$ to $L_{G}=5 \mathrm{~nm}$ and the rest of the parameters remain constant: the gate oxide with Equivalent Oxide Thickness $E O T=1 \mathrm{~nm}$, and the gate work function of $4.385 \mathrm{eV}$. The Si thickness is $3 \mathrm{~nm}$ and abrupt doping profiles in the S/D-channel junctions with $\mathrm{N}_{D}=1 \times 10^{20} \mathrm{~cm}^{-3}$ have been considered.

Our simulation frame will only consider ballistic transport and S/D tunneling considering two approaches: ballistic tunneling (BT) where particles are drifted inside the barrier region as previously described [9] and instantaneous tunneling (IT), a simpler model which does not consider the transport inside the barrier with two implications: the tunneling time is zero and no charge is considered under the barrier affecting to the self consistent solution of Poisson equation. The differences between the models are clearly presented in Figure 3. In this case, a $5 \mathrm{~nm}$ gate length device is considered. Instantaneous tunnel presents an electron distribution similar to the expected in the case of classical transport with no quantum effects, Figure 3 (top). No charge is considered inside the barrier, as a consequence, there is no important effect on subband profile as shown in Figure 4. However, Figure 3 (bottom) includes the effect of transport inside the barrier. This fact affects the subband distribution obtained from Poisson and Schrödinger equations. As observed in Figure 4, the first subband approaches to the obtained with NEGF codes.

Previous plots correspond to subthreshold conditions. Therefore, the charge due to thermionic emission is very small. As a consequence, an important fraction of the charge in the channel comes from the tunneling. Figure 5 shows the inversion charge in the $\mathrm{L}_{G}=5 \mathrm{~nm}$ device under the same conditions already discussed. It can be observed that IT charge is very similar to the calculations obtained from a pure semiclassical transport model with no tunneling. However, BT model reproduces the charge obtained with NEGF calculations highlighting the importance of consider the contribution of tunneling electrons not only to the transport but to the electrostatics. It is also interesting to remark, that charge profile is practically unaltered for all the MS-EMC calculations outside the tunneling area. As observed in Figure 5, for $X=-2 n m$ the BT curve splits from the others following the NEGF profile, indicating the contribution of charge under the barrier with a good agreement with the quantum transport calculations. 


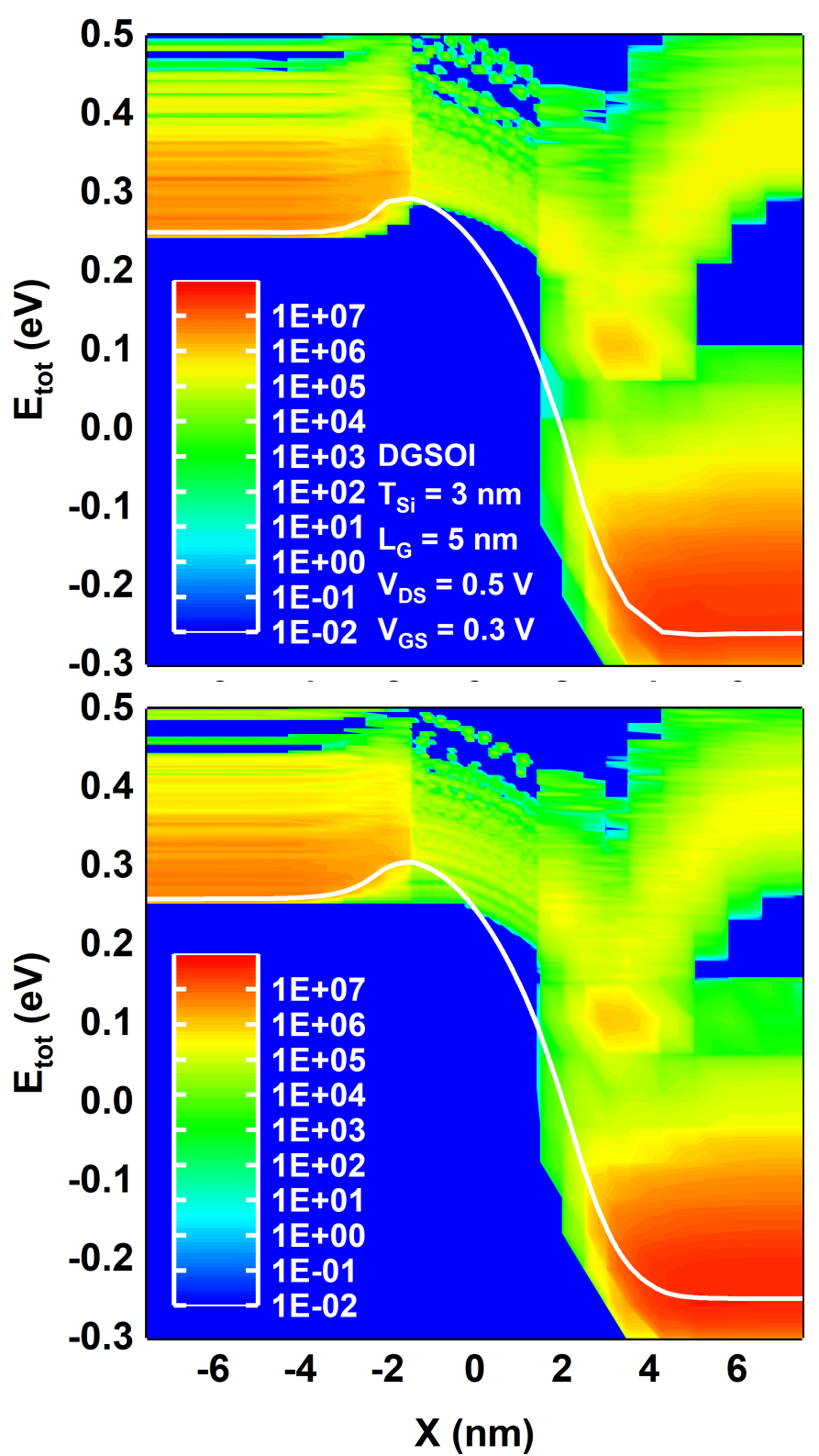

Fig. 3. Electron distribution in the first subband as a function of the position and the total energy for a DGSOI device with $\mathrm{L}_{G}=5 \mathrm{~nm}, \mathrm{~T}_{S i}=3 \mathrm{~nm}$, $\mathrm{V}_{G}=0.3 \mathrm{~V}$ and $\mathrm{V}_{D S}=0.5 \mathrm{~V}$ considering instantaneous tunneling (top) and ballistic (bottom) models.

The $\mathrm{I}_{D}-\mathrm{V}_{G}$ characteristics for a $7.5 \mathrm{~nm}$ and $5 \mathrm{~nm}$ gate length transistors are shown in Figure 6 top and bottom respectively. For all the cases, a very good agreement is observed in the $\mathrm{ON}$ region whereas the subthreshold region presents some differences. Concerning the high gate voltage conditions, the tunnel probability given by WKB is very high since the barrier decreases as we increase $\mathrm{V}_{G}$. However, this also increases the thermionic emission and, therefore, the fraction of electrons that contribute to the current is very small compared to those coming from thermionic emission. It is interesting to highlight the importance of the tunneling model in the subthreshold region. When IT is considered, there is a small difference

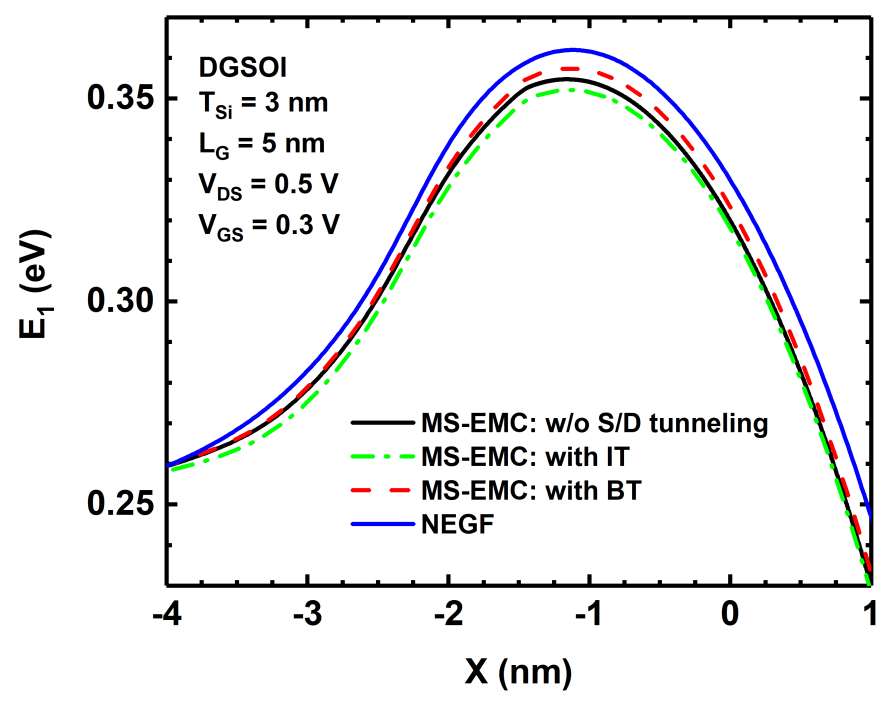

Fig. 4. Energy profile of the lowest energy subband in the 5nm DGSOI device at $\mathrm{V}_{D S}=0.5 \mathrm{~V}$ and $\mathrm{V}_{G S}=0.2 \mathrm{~V}$ for the NEGF tool as well as the MS-EMC considering a simulation w/o S/D tunneling and both the instantaneous and the ballistic S/D tunneling models.

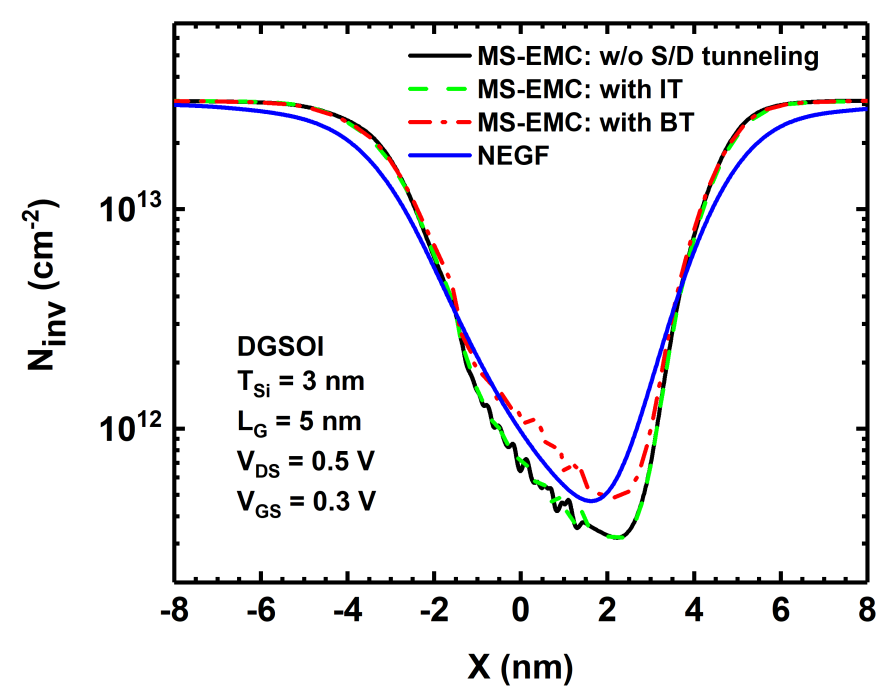

Fig. 5. Electron distribution in the $5 \mathrm{~nm}$ DGSOI device at $\mathrm{V}_{D S}=0.5 \mathrm{~V}$ for the NEGF tool as well as the MS-EMC considering a simulation w/o S/D tunneling and both the instantaneous and the ballistic S/D tunneling models. $\mathrm{V}_{D S}=0.5 \mathrm{~V}$ and $\mathrm{V}_{G S}=0.2 \mathrm{~V}$.

with the simulations that do not take tunneling into account. However, the inclusion of BT degrades the subthreshold characteristics approaching closely the NEGF results. This trend can be observed when moving from $7.5 \mathrm{~nm}$ to $5 \mathrm{~nm}$ devices where NEGF and MS-EMC with BT perfectly match.

\section{CONCLUSIONS}

This work presents the implementation of S/D tunneling in a MSB-EMC tool and its comparison to NEGF approaches. Instantaneous and ballistic tunneling approaches have been considered to describe the behavior of electrons inside the forbidden region. Our simulations show a better agreement 


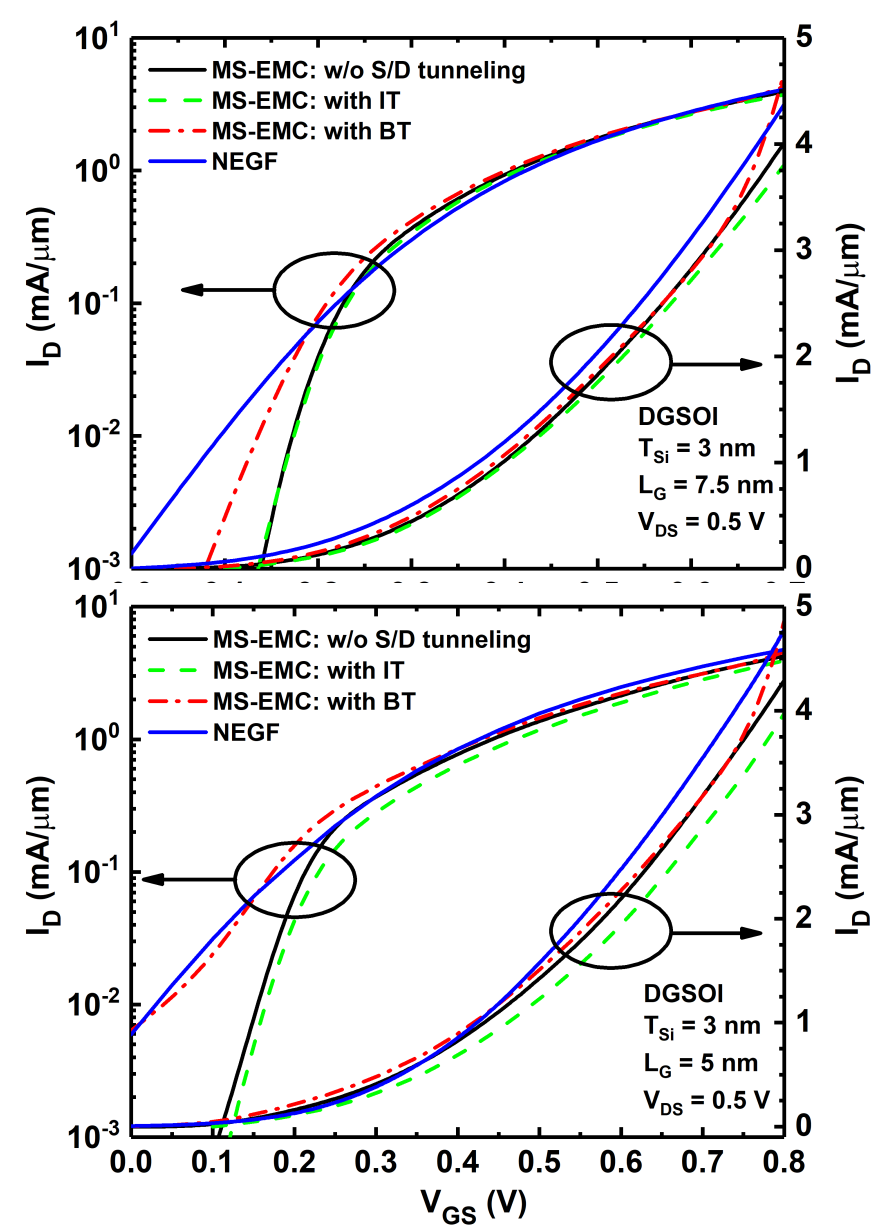

Fig. 6. $\mathrm{I}_{D}$ vs. $\mathrm{V}_{G S}$ in the $7.5 \mathrm{~nm}$ (top) and $5 \mathrm{~nm}$ DGSOI devices at $\mathrm{V}_{D S}=0.5 \mathrm{~V}$ for the NEGF tool as well as the MS-EMC considering a simulation without S/D tunneling and both the instantaneous and the ballistic S/D tunneling models.

using BT models since the degradation in subthreshold region and the charge inside the barrier correspond to the obtained with NEGF codes. Once the model has been calibrated on the ballistic regime, the MS-EMC simulator will be an useful tool for the optimization of devices targeting sub-10 nm nodes thanks to its higher efficiency from a computational point of view when scattering is included.

\section{ACKNOWLEDGMENT}

The authors are grateful for the support given by the Spanish Ministry of Economy, Industry and Competitivity (TEC2014-59730-R and TEC2017-89800-R), H2020 - REMINDER (687931) and H2020 - WAYTOGO-FAST (662175).

\section{REFERENCES}

[1] H.-S. Wong, "Beyond the conventional transistor," IBM Journal of Research and Development, vol. 46, pp. 133-168, March 2002.

[2] K. Roy, S. Mukhopadhyay, and H. Mahmoodi-Meimand, "Leakage Current Mechanisms and Leakage Reduction Techniques in DeepSubmicrometer CMOS Circuits," Proceedings of the IEEE, vol. 91, pp. 305-327, February 2003.

[3] J. W. J. Wang and M. Lundstrom, "Does source-to-drain tunneling limit the ultimate scaling of MOSFETs?" Digest. International Electron Devices Meeting, pp. 707-710, 2002.

[4] I. Hiroshi, "Future of nano CMOS technology," Solid-State Electronics, vol. 112, pp. 56-67, March 2015.

[5] C. Sampedro, F. Gámiz, A. Godoy, R. Valín, A. García-Loureiro, and F. G. Ruiz, "Multi-Subband Monte Carlo study of device orientation effects in ultra-short channel DGSOI," Solid-State Electronics, vol. 54 no. 2, pp. 131-136, 2010.

[6] C. Sampedro, F. Gámiz, and A. Godoy, "On the extension of ET-FDSOI roadmap for $22 \mathrm{~nm}$ node and beyond," Solid-State Electronics, vol. 90, pp. 23-27, 2013.

[7] R. Venugopal, Z. Ren, S. Datta, M. S. Lundstrom, and D. Jovanovic, "Simulating quantum transport in nanoscale transistors: Real versus mode-space approaches," Journal of Applied Physics, vol. 92, no. 7, pp. 3730-3739, 2002.

[8] C. Medina-Bailon, C. Sampedro, F. Gámiz, A. Godoy, and L. Donetti, "Confinement orientation effects in S / D tunneling," Solid-State Electronics, vol. 128, pp. 48 - 53, 2017.

[9] C. Medina-Bailon, T. Sadi, C. Sampedro, J. Padilla, A. Godoy, L. Donetti, V. Georgiev, F. Gamiz, and A. Asenov, "Assessment of gate leakage mechanism utilizing multi-subband ensemble monte carlo," in 2017 Joint International EUROSOI Workshop and International Conference on Ultimate Integration on Silicon (EUROSOI-ULIS). IEEE, apr 2017.

[10] C. Medina-Bailon, J. L. Padilla, C. Sampedro, C. Alper, F. Gamiz, and A. M. Ionescu, "Implementation of band-to-band tunneling phenomena in a multisubband ensemble monte carlo simulator: Application to silicon TFETs," IEEE Transactions on Electron Devices, vol. 64, no. 8, pp. 3084-3091, aug 2017.

[11] C. Shen, L.-T. Yang, G. Samudra, and Y.-C. Yeo, "A new robust nonlocal algorithm for band-to-band tunneling simulation and its application to Tunnel-FET," Solid-State Electronics, vol. 57, no. 1, pp. 23-30, Mar. 2011.

[12] D. J. Griffiths, “The WKB approximation,” in Introduction to Quantum Mechanics. New Jersey: Prentice Hall, 1995, ch. 8, pp. 274-297.

[13] A. Revelant, P. Palestri, and L. Selmi, "Multi-subband semi-classical simulation of n-type Tunnel-FETs," in 2012 13th International Conference on Ultimate Integration on Silicon (ULIS). Ieee, Mar. 2012, pp. 187-190.

[14] Z. Huang, T. E. Feuchtwang, P. H. Cutler, and E. Kazes, "WentzelKramers-Brillouin method in multidimensional tunneling," Physical Review A, vol. 41, no. 1, pp. 32-41, 1990.

[15] M. Lundstrom, "Nanomos," May 2006. [Online]. Available: https: //nanohub.org/resources/nanomos 